\title{
Tonometry and Intraocular Pressure - Where are we Now?
}

\author{
Julian García-Feijoo
}

Professor and Chairman of Ophthalmology, Department of Ophthalmology, Hospital Clínico San Carlos; Universidad Complutense de Madrid, Instituto de Investigación Sanitaria del Hospital Clínico San Carlos (IdISSC), Madrid, Spain; Cooperative Research Network on Age-Related Ocular Pathology, Visual and Life Quality, Instituto de Salud Carlos III, Madrid, Spain

\begin{abstract}
Goldmann applanation tonometry has been the gold standard for measuring intraocular pressure for many years. However, it has some limitations, including the effects of several ocular variables such as axial length, curvature, rigidity and corneal thickness on the measurements. These limitations have prompted the development of new tonometers.

\section{Keywords}

Glaucoma, intraocular pressure, tonometry, rebound tonometer, dynamic contour tonometer, ocular response analyzer

Disclosure: Julian García-Feijoo has received Clinical Trials/Research Support to his institution from SENSIMED and Tiolat. No funding was received in the publication of this article. This article is a short opinion piece and has not been submitted to external peer reviewers but was reviewed by the advisory board before publication.

open Access: This article is published under the Creative Commons Attribution Noncommercial License, which permits any noncommercial use, distribution, adaptation, and reproduction provided the original author(s) and source are given appropriate credit.

Received: 14 July 2016 Pulished Online: 4 August 2016 Citation: European Ophthalmic Review, 2016;10(1):22-4

Correspondence: Julian García-Feijoo, Paseo de San Francisco de Sales 23, Casa 2,10B, 28003 Madrid, Spain. E: jgarciafeijoo@hotmail.com
\end{abstract}

Glaucoma is an optic neuropathy, and although considered a multifactorial disease, it is the level of intraocular pressure (IOP) that is the main known risk factor for development and progression. There are other risk factors that can modify the effect of IOP on the development of glaucoma, but currently the main objective of glaucoma management is to preserve visual function by achieving a stable and continuous IOP decrease. Therefore, even if there are other factors to consider in glaucoma besides IOP, its assessment plays a fundamental role in the evaluation of the risk of conversion and progression.

Goldmann applanation tonometry (GAT) is the gold standard for measuring IOP and most probably will continue to be so into the future. However, its limitations were obvious from the start and include the substantial effects of several eye variables such as axial length, curvature, rigidity and corneal thickness. Also, in some eyes, especially those with corneal problems (such as diseases, trauma, haze, corneal opacities, leucomas, etc.) and surgery, taking measurements is not easy.

These limitations have prompted the development of new tonometers to improve the measurement of IOP. However, other pending questions exist, such as, which aspects of the IOP should be considered, and how do they interact with other factors? It has been clearly established that single snapshot IOP measurements do not provide enough information on the dynamic behaviour of the IOP. In fact, glaucoma is a 24-hour disease and the IOP exerts its effect on the ocular structures (including the optic nerve head, trabecular meshwork, etc.) all day long, on a daily basis. So, not only do we need to consider IOP variables such as fluctuation, peak pressure, IOP range, but also how these variables may impact eyes with different biomechanical and structural properties and also its complex interaction with other pressures (intracranial, translaminar, blood pressure, etc.).
Therefore, we need to start looking at IOP in a different way. IOP is a dynamic variable that fluctuates throughout a 24-hour period, yet patient follow-up is generally based on isolated IOP readings. It is true that we can adequately assess and manage the majority of glaucoma patients with single IOP measurements using GAT. However, one could question, is this really good enough, or could we do any better? The main challenges include: obtaining IOP measurements not influenced by the corneal properties; to develop technologies to measure the circadian IOP in clinical practice; and to understand better the complex interactions between IOP, other pressures and ocular biomechanics so we can effectively use the most relevant parameters to characterise the disease and improve patient care.

Rebound tonometry (Icare ${ }^{\circledR}$ and Icare ${ }^{\circledR}$ PRO; Icare, Oy, Helsinki, Finland), is based on the induction rebound principle, but measurements obtained with it are affected by corneal thickness. ${ }^{1,2}$ However, a clear advantage of the Icare PRO over the other four iCare models is that measurements may be taken with the patient lying or sitting/ standing. Also the small probe size of these tonometers facilitates its use in children and in eyes with corneal abnormalities and make it easier to measure IOP after corneal grafts, corneal rings, etc., as we can select the best area to measure. Therefore, although it is not a breakthrough, it addresses some practical problems of applanation tonometry. However, we also have to consider the increased cost of having to use disposable probes.

Dynamic contour tonometer (DCT), or Pascal tonometer (SMT Swiss Microtechnology AG, Port, Switzerland) has a $7 \mathrm{~mm}$ diameter concavesurface probe that adapts to the cornea's contour and does not alter its shape or curvature. An electronic pressure sensor embedded in the tonometer's concave probe surface enables the direct measurement of transcorneal pressure. Such measurements are, at least a priori, 
Table 1: Overview of tonometers and their advantages and disadvantages

\begin{tabular}{|c|c|c|}
\hline Tonometer & Advantages & Disadvantages \\
\hline $\begin{array}{l}\text { Rebound tonometry (Icare and } \\
\text { Icare PRO) }\end{array}$ & $\begin{array}{l}\text { - Icare PRO allows measurements to be taken with the } \\
\text { patient lying or sitting/standing } \\
\text { - Small size of probes allows it to be used in children and } \\
\text { allows the best area of measurement to be selected in } \\
\text { eyes with corneal abnormalities }\end{array}$ & $\begin{array}{l}\text { - Measurements obtained are affected by corneal thickness } \\
\text { - The use of disposable probes increase the cost }\end{array}$ \\
\hline $\begin{array}{l}\text { Dynamic contour tonometer or } \\
\text { Pascal tonometer }\end{array}$ & $\begin{array}{l}\text { - Measures transcorneal pressure } \\
\text { - Most independent tonometer of corneal thickness } \\
\text { - Best option after corneal refractive laser }\end{array}$ & $\begin{array}{l}\text { - Remember that IOP readings are higher than with GAT } \\
\text { - } \text { Cost of the probe cover } \\
\text { - Measurement takes time }\end{array}$ \\
\hline Ocular Response Analyzer & $\begin{array}{l}\text { - Adds biomechanical Data } \\
\text { - Hysteresis and corneal resistance } \\
\text { - Useful for research }\end{array}$ & $\begin{array}{l}\text { - } \text { Cost of device } \\
\text { - Real meaning of corrected IOP to be established }\end{array}$ \\
\hline Corvis ST & $\begin{array}{l}\text { - Adds biomechanical Data } \\
\text { - Variables related to corneal deformability } \\
\text { Useful for research }\end{array}$ & $\begin{array}{l}\text { - } \text { Cost } \\
\text { biomechanical variables }\end{array}$ \\
\hline Triggerfish & - Provides 24-hour data & $\begin{array}{l}\text { - Does not measure IOP } \\
\text { - Cost } \\
\text { - Antenna is worn as a ring around the orbit } \\
\text { - Side effects of hypermia and punctate keratitis very common }\end{array}$ \\
\hline
\end{tabular}

GAT = Goldmann applanation tonometry; IOP = intraocular pressure.

independent of corneal properties. In fact, most studies published to date concur in that DCT is perhaps currently the tonometer that shows most independence of corneal thickness..$^{3-5}$ Obviously, there are measurement differences noted with the GAT (mean overestimation $2.3 \mathrm{mmHg}$ ) so the two tonometers may not be interchangeably used. However, the DCT can provide valuable information in persons with a corneal thickness far from the mean or in patients who have undergone refractive surgery. ${ }^{6}$ Again, disposable covers are needed and due to the time needed to complete the measurement, it is also necessary to have strong patient collaboration to obtain reliable measurements. Also, taking measurements in challenging corneas (such as extreme curvature or corneal surface irregularities, corneal grafts, corneal rings, etc.) is not easy, and often the measurements are not reliable in these cases.

The Ocular Response Analyzer ${ }^{\circledast}$ (ORA) (Reichert Inc., Depew, New York, US) and Corvis ${ }^{\circledR}$ ST (Optikgeräte GmbH, Oculus, Wetzlar, Germany) tonometers are a step forward, and not only measure the IOP, but also provide information on the biomechanical properties of the cornea and thus have the potential to correct IOP readings for these factors. Furthermore, there is the option to independently analyse some corneal biomechanical variables and even dynamic corneal behaviour. All of these can be correlated with other ocular biomechanical variables and eventually could help us to understand some of the complex physiopathology of glaucoma.

The ORA is an airpulse tonometer and introduces two new concepts relating to the deformability of the cornea: corneal hysteresis and corneal resistance. ${ }^{7.8}$ The corneal hysteresis reflects the capacity of the cornea to absorb and dissipate energy and forms the basis for the instrument's correction of IOP. The result of this correction is known as compensated IOP (IOPC). This new pressure should be, at least in theory, less affected by corneal properties than that provided by a conventional applanation tonometer. The corneal resistance factor indicates the overall resistance exerted by the cornea and is related to central corneal thickness and IOP. Whilst there is promising published evidence, the role of corneal hysteresis and the corneal resistance factor in the diagnosis and management of patients with glaucoma is yet to be fully established.

The Corvis ST is an air pulse tonometer and in-built pachymeter that also provides measurements of corneal biomechanical properties. ${ }^{9}$ The instrument incorporates an ultra high-speed Scheimpflug camera and records the anterior chamber at the moment of corneal deformation in real time. It measures variables related to corneal deformability such as the time, velocity and length of the first and second applanations, maximal concavity, and deformation amplitude. Further work is needed to identify the biomechanical variables measured by the Corvis ST that could be related to the development and progression of glaucoma. Whilst this is an interesting device, one potential disadvantage is the cost.

The question of developing an easy and cheap method to obtain 24-hour IOP measurements remains unsolved. Although some intraocular sensors are under development, there is one commercially available contact lens that allows for the assessment of the IOP. This device, the Triggerfish ${ }^{\circledR}$ (Sensimed AG, Lausanne, Switzerland), consists of a silicone contact lens with an embedded pressure sensor that enables IOP monitoring while patients continue with their routine daily activity, in fact the sensor takes pressure measurements over 30 seconds every five minutes to provide 288 measurements after 24 hours of lens wear. ${ }^{10,11}$ The device does not measure 'real' IOP but its sensor converts the electrical signal obtained into a tension curve expressed in arbitrary units so that it can help to determine the circadian pattern of the IOP. However, more work is needed to understand how these measurements can be applied to real life clinical practice. ${ }^{12}$ Some other disadvantages are the cost, problems related to wearing the device for 24 hours (such as hyperemia, punctate keratitis) and also the patient has to wear an antenna that transmits the data as a ring around the orbit.

Home tonometry is another option and the Icare ${ }^{\circledR}$ ONE (iCare Finland, Oy, Finland) and Icare HOME (iCare Finland, Oy, Finland) have been specifically designed for this purpose so that it can easily be used by patients. However, the learning curve of use is influenced by 


\section{Editorial Glaucoma}

age and social level of the patient. There are other options, such as transpalpebral tonometers, but they lack reliability and the correlation with applanation tonometry is very poor.

\section{Conclusions}

We are hopeful that, in the future, we will be able to use devices with sensors that will provide us with accurate and complex information on the dynamic behaviour of IOP. Moreover, we will have access to additional information on ocular biomechanical properties which will probably help us to better evaluate and understand the different susceptibility to IOP-related damage. In the future, our work will be to filter all this additional information and determine what parameters are most relevant to improve the clinical management of our glaucoma patients. [
1. Kontiola Al, A new electromechanical method for measuring intraocular pressure, Doc Ophthalmol, 1997;93:265-76.

2. Martinez-de-la-Casa JM, Garcia-Feijoo J, Castillo A, GarciaSanchez J, Reproducibility and clinical evaluation of rebound tonometry, Invest Ophthalmol Vis Sci, 2005;46:4578-80.

3. Kaufmann C, Bachmann LM, Thiel MA, Comparison of dynamic contour tonometry with Goldmann applanation tonometry Invest Ophthalmol Vis Sci, 2004:45:3118-21.

4. Kotecha A, White ET, Shewry JM, Garway-Heath DF, The relative effects of corneal thickness and age on Goldmann relative effects of coneal thickness and age on Goldmann applanation tonometry and dynamic

5. Martinez-de-la-Casa JM, Garcia-Feijoo J, Vico E, et al., Effect Martinez-de-la-Casa JM, Garcia-Feijoo J, Vico E, et al., Effect
of corneal thickness on dynamic contour, rebound, and
Goldmann tonometry, Ophthalmology, 2006;113:2156-62 6. Pepose JS, Feigenbaum SK, Qazi MA, et al., Changes in corneal biomechanics and intraocular pressure following LASIK using static, dynamic, and noncontact tonometry, Am J Ophthalmol, 2007;143:39-47.

7. Luce DA, Determining in vivo biomechanical properties of the cornea with an ocular response analyser, $J$ Cataract Refract Surg, 2005;31:156-62.

8. Martinez-de-la-Casa JM, Garcia-Feijoo J, Fernandez-Vidal A, et al., Ocular response analyzer versus Goldmann applanation tonometry for intraocular pressure measurements, Invest Ophthalmol Vis Sci, 2006;47:4410-4

9. Hong J, Xu J, Wei A, et al., A new tonometer-the Corvis ST tonometer: clinical comparison with noncontact and
Goldmann applanation tonometers, Invest Ophthalmol Vis Sci, 2013;54:659-65.

10. Leonardi M, Leuenberger P, Bertrand D, et al., First steps toward noninvasive intraocular pressure monitoring with a sensing contact lens, Invest Ophthalmol Vis SCi, 2004:45:3113-7.

11. De Smedt S, Mermoud A, Schnyder C, 24-hour intraocular pressure fluctuation monitoring using an ocular telemetry sensor: tolerability and functionality in healthy subjects, J Glaucoma, 2012;21:539-44.

12. Mansouri K, Medeiros FA, Tafreshi A, Weinreb RN, Continuous 24-hour monitoring of intraocular pressure patterns with a 24-hour monitoring of intraocular pressure patterns with a
contact lens sensor: safety, tolerability, and reproducibility in contact lens sensor: safety, tolerability, and reproducibility in
patients with glaucoma, Arch Ophthalmol, 2012;130:153-9. 